LBL-36219

UC-414

Invited paper presented and to be published in the Proceedings of the NATO Advanced Research Workshop,

"Hot Hadronic Matter: Theory and Experiment",

Divonne-les-Bains, France, June 27-July 1, 1994, Plenum Press Publishing Co.

Similarities and Differences in Strangeness Production at BNL and CERN

\author{
Grazyna Odyniec \\ Lawrence Berkeley Laboratory \\ University of California, Berkeley, CA 94720
}

September 1994

This work was supported by the Director, Office of Energy Research, Division of Nuclear Physics of the Office of High Energy and Nuclear Physics of the U.S. Department of Energy under Cortract DEAC03-76SF00098 


\title{
SIMILARITIES AND DIFFERENCES IN STRANGENESS PRODUCTION AT BNL AND CERN
}

\author{
Grazyna Odyniec \\ Lawrence Berkeley Laboratory \\ University of California \\ Berkeley, CA 94720
}

\begin{abstract}
Relativistic heavy ion collisions provide, in principle, necessary conditions for the formation of the quark gluon plasma. Strangeness enhancement was amoung the announced specific signals expected from this new, hypothetical state of nuclear matter. Analysis of the first generation of experiments with light and medium sized ions have been completed. Results on strangeness production become precious handles to study in great detail what is actually happening. The current experimental situation is assessed. The emerging picture is still incomplete: however, open questions constrain requirements on future heavy ion experiments.
\end{abstract}

\section{INTRODUCTION}

The search for a quark-gluon plasma has been a long one. The creation of the primordial quark-gluon plasma phase in laboratories and subsequent study of its properties has been an ultimate goal for nuclear scientists since the mid-seventies. Thanks to Quantum Chromodynamics, we know what this new state is likely to be. We know rather precisely the value of the key parameters, such as the temperature and the baryon chemical potential, beyond which nuclear matter can only exist in the form of quarks and gluons in thermal equilibrium. Furthermore, we also know what kind of characteristic signals should mark the formation of a new plasma phase. Strangeness is one of them. Enhancement of strange particle production in the plasma phase as compared to a thermalized hadronic gas, was originally proposed by Koch, Muller and Rafelski ${ }^{1}$ almost a decade ago. Indeed, high quark and gluon densities and frequent partonic collisions together with a temperature on the order of $200 \mathrm{MeV}$ (which should a priori correspond to the plasma phase) should enhance the strangeness content. of the final state with insufficient time to retum to equilibrium in a hadronic phase at lower temperature.

But achieving all the prerequisite conditions experimentally may still not be enough for the formation of a quark-gluon plasma phase in the laboratory. Perhaps there is no simple way to detect it as well. Nevertheless, much can be learned about this new form of highly excited nuclea: matter by studying strange particles in general, and multistrange baryons in particular.

In this lecture, the current status of selected experimental results on strangeness production in relativistic ion collisions at CERN and BNL is reviewed. I will try to 
convey not only my own understanding of the present experimental situation, but also those arising from discussions which took place during this meeting. I would like to apologize in advance to those who may feei that their results are omitted or not fairly represented here. It is clear that due to the time constraints I have had to make choices, emphasizing more the data which are suitable for the comparisons between the two energy regimes.

\section{"FAVORABLE" CONDITIONS AT CERN AND AGS FOR QGP FORMATION}

Transition to the hypothetical quark-gluon plasma phase might only take place when the energy density and temperature, reached in the early stages of the collision, exceed their critical values. Creation of a high density requires large energy deposition from the longitudinal motion of the projectile into the interaction volume. In the early eighties, it was realized that there was a chance to reach at least the energy density needed with collisions of heavy ions accelerated in existing machines. Soon after, an exploratory program was successfully launched at the Brookhaven AGS with silicon beams at 14 $\mathrm{GeV} / \mathrm{c}$ and at the CERN SPS with oxygen and sulphur beams at $200 \mathrm{GeV} / \mathrm{c}$.

The first results were as expected: a significant degree of stopping appeared at both energies. This was the first piece of good news, especially in case of the CERN data, since a rather large degree of transparency was forecast for such a high energy domain, which would, undoubtedly, complicate the interpretation of the data.

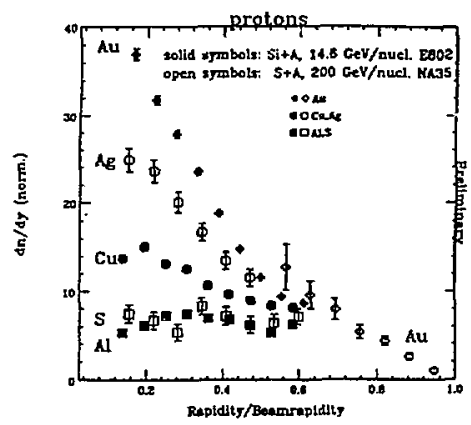

Figure 1: Relative rapidity ( $y /$ /ybeam) distributions of primordial protons in nucleus-nucleus collisions from the AGS E802 experiment (solid symbols) and the CERN NA35 experiment (open symbols).

Fig.1 shows the compilation of the relative rapidity distributions ( $y / y_{\text {beam }}$ ) of net protons for various nucleus-nucleus collisions observed at both energies. Solid symbols represent AGS E802 experiments at $14.6 \mathrm{GeV} / \mathrm{c}$, and an open symbols - CERN NA35 data $^{a}$ at $200 \mathrm{GeV} / \mathrm{c}$. First of all, we see that in all reactions there is an appreciable number of baryons at mid-rapidity, and that with increasing target mass more and more projectile and target nucleons are shifted towards mid-rapidity. Momentary stopping of projectiles on target nucleons implies a momentary high baryon energy density. Thus the high energy density seems to be well within reach in experiments at both laboratories.

\footnotetext{
a Since the streamer chamber (NA35 CERN experiment) has no identification capability for stable particles the net proton distribution was deduced from the measurement of the charge excess by subtracting negative hadron distributions from positive hadron distributions and applying all the necessary corrections.
} 
Th: second piece of good news came in 1988 from the NA35 experiment at CERN and the E802 experiment at BNL.

NA35 observed for the first time a significant enhancement ( factor 2$)$ of $\Lambda, \bar{\Lambda}$ and $\mathrm{K}^{\circ}$ particle production in $\mathrm{S}+\mathrm{S}$ collisions at $200 \mathrm{GeV}^{2}$. Initial estimates of energy densities in central S+S collisions ${ }^{3}$ were of the order of $2 \mathrm{GeV} / \mathrm{fm}^{3}$ and appeared to be, in principle, adequate for plasma formation. However, this was not likely to happen due to the rather small volume $\left(-90 \mathrm{fm}^{3}\right)$ which did not seem to be sufficient even for partial thermalization.

Also, the BNL E802 collaboration reported an enhanced $\mathrm{K}^{+} / \pi^{+}$ratio in $\mathrm{Si}+\mathrm{Au}$ collisions at $14.6 \mathrm{GeV}^{4}$.

This initial excitement stimulated a lot of experimental and theoretical effort. The data, available now, are more mature and better understood; however, theory still does not offer an explanation of the observed phenomena.

\section{RECENT AGS RESULTS ON STRANGENESS}

The E859 experiment was designed to improve and expand on single particle inclusive and two-particle correlation data obtained previously by the E802 collabotation. Single particle inclusive measurements have been made for the wealth of particle species, as illustrated in Fig.2. The E859 data analysis provided integrated yields, dN/dy, for both $\mathrm{K}^{+}$and $\mathrm{K}^{-}$in several ranges of rapidities, different centralities and targets. In turn, these results were used to investigate whether the $\mathrm{K}^{+} / \pi^{+}$enhancement reported earlier by the E802 experiment also persisted in the $\mathrm{K}^{-}$channel.

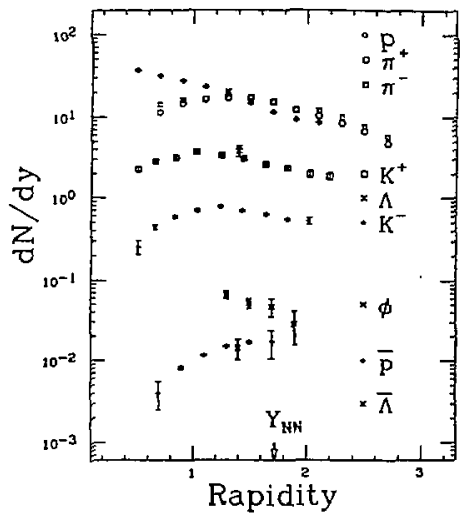

Figure 2: Rapidity distributions for all the particle species measured by the E802 and E859 experiments. Values shown are for central Si+Au collisions at $14.6 \mathrm{GeV} / \mathrm{c}$.

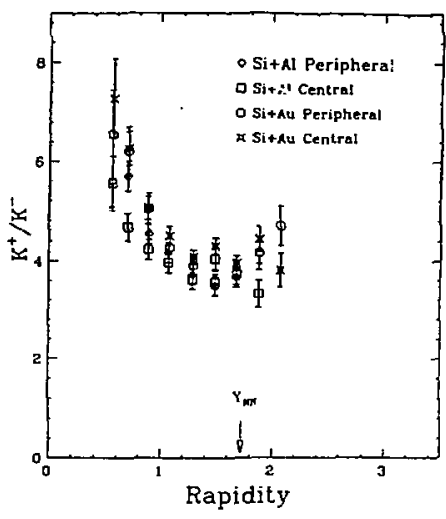

Figure 3: $\mathrm{K}^{+} / \mathrm{K}^{-}$ratios vs. rapidity for peripheral and central $\mathrm{Si}+\mathrm{Al}$ and $\mathrm{Si}+\mathrm{Au}$ collisions from $\mathrm{E} 859$ experiment at AGS.

Fig. 3 shows the $\mathrm{K}^{+} / \mathrm{K}^{-}$ratios as a function of rapidity for peripheral and central $\mathrm{Si}+\mathrm{Al}$ and $\mathrm{Si}+\mathrm{Au}$ collisions. In both reactions, the $\mathrm{K}^{-}$and $\mathrm{K}^{+}$yields scale almost exactly with each other. Both the magnitude and rapidity dependence remain unchanged. However, the absolute number of kaons in these two reactions differs by more than a factor of 25 and the $K / \pi$ ratio by a factor of 2 . The fact that the enhancement of $K+$ and 
$\mathrm{K}^{-}$seems to be the same, even though the particles are believed to have very different production mechanisms, will require special attention within the theoretical models.

Furthermore, the $\mathrm{K}^{+} / \pi^{+}$signal may allow for the assessment of the degree of thermalization. The increase of the $\mathrm{K}^{+} / \pi^{+}$ratio with the incident ion size is illustrated in Fig.4.

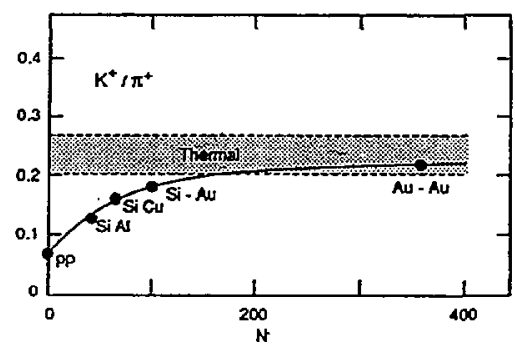

Figure 4: $\mathrm{K}^{+} / \pi^{+}$ratio versus incident ion size at AGS energies.

It gradually rises by a factor of two from p-p to A-A collisions and then levels off. A thermal system at a temperature of about $200 \mathrm{MeV}$ is expected to favor far more kaons as compared to pions than the typical p-p collision $\left[m_{t}(K) \sim m_{t}(\pi)\right]$. However, one is rather surprised to see the symptoms of thermalization already at the AGS energies since this energy is believed to be too low for thermalization ${ }^{5}$.

Recently the E859 collaboration reported measurements of $\Lambda$ and $\bar{\Lambda}$. Earlier measurements of $\Lambda$ production by the E810 experiment were confirmed and expanded, and the first results on $\bar{\Lambda}$ production (one-point, spectrometer integrated yield for central $\mathrm{Si}+$ Au collision at mid-rapidity, shown in Fig.2) on a heavy target were obtained. The data with an inverted magnetic field to collect lambdas with the same acceptance as $\Lambda$ were used to extract a ratio. At the moment the preliminary value of the $\Lambda / \Lambda$ ratio is estimated to be about $(4.0 \pm 2) \times 10^{-3}$ with large systematic uncertainty. Details of the analysis and some very interesting results on antilambda/antiproton ratios are discussed by S.G.Steadman in these proceedings.

The first observation of multistrange baryon production in heavy ion collisions at the AGS was made by E810 collaboration ${ }^{6}$. The lifetime of $\Xi^{-}$measured in $\mathrm{Si}$ on $\mathrm{Pb}$ collisions at $14.6 \mathrm{GeV} / \mathrm{c}$ agrees very well with the particle data group value. Fig.5 presents the observed $\Xi^{-}$effective mass spectrum (a) and the rapidity distribution (b) after an acceptance correction.
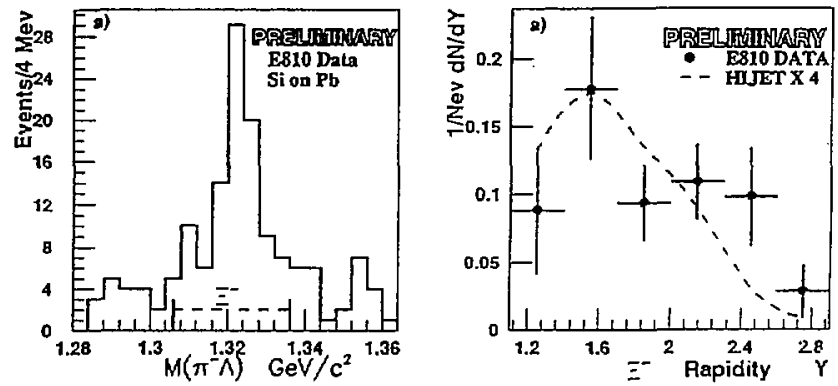

Figure 5: $\Xi$ - effective mass spectrum (a) and the rapidity distribution (b) in Si on Pb collisions at 14.6 $\mathrm{GeV} / \mathrm{c}$ (E810 experiment). 
Some qualitative description of the AGS experimental results was provided by the ARC, a relativistic particle cascade model, without need for any novel mechanism for enhanced strangeness production. The ARC simulations indicate transient presence of a clearly defined high density matter at mid-rapidity. This suggests that the local equilibrium thermal model (fireball) may provide the correct description of the collision. Such an approach was advocated by Rafelski and Danos ${ }^{7}$, and with further development by Lettesier, Rafelski and Tounsi ${ }^{8}$ to extract chemical potential values from the available experimental data. The basic difference between this method and other models ${ }^{9}$ is that instead of testing the validity of a particular model, they developed a method of translating the experimental results into a set of parameters characterizing the collision. Under very few, but rather strong, general assumptions these parameters can be interpreted as the usual thermal parameters (temperature and chemical potentials) of the hadron system at freeze-out ${ }^{10}$. They assume that all central rapidity strange particles are emitted from a thermally equilibrated fireball with $u$ and $d$ flavors in chemical equilibrium, but the strange quark flavor only in relative chemical equilibriumb. As a result of this approximation the relative yields of the different particles are described in terms of two fugacity parameters ${ }^{c}$ : the strange-quark fugacity $\lambda_{s}$ and the light-quark fugacity $\lambda_{\mathrm{q}}$. Namely:

$$
\bar{N} \Lambda=\lambda_{s}{ }^{-2} \lambda_{q}{ }^{4} \quad \text { and } \quad K+/ K^{-}=\lambda_{s}{ }^{-2} \lambda_{q}{ }^{2} \text {. }
$$

These permit the determination of fugacity values and chemical potentials from experimental particle yields. The E859 obtained yields with $\mathrm{Si}+\mathrm{Au}$ at $14.6 \mathrm{GeV} / \mathrm{c}$, even though the data is still preliminary, of :

$$
\begin{gathered}
\bar{N} \Lambda \sim(4 \pm 2) \times 10^{-3} \text { (in the central rapidity region, see Fig.2), and } \\
\mathrm{K}^{+} / \mathrm{K}=4.5 \pm 0.5 \begin{array}{l}
\text { (which is constant in the entire central rapidity range } \\
1<y<1.6, \text { but varies strongly near target and projectile } \\
\text { rapidities (see Fig.3)) }
\end{array}
\end{gathered}
$$

ase presented graphically in the $\lambda_{q}$ vs. $\lambda_{s}$ plane in Fig.6.

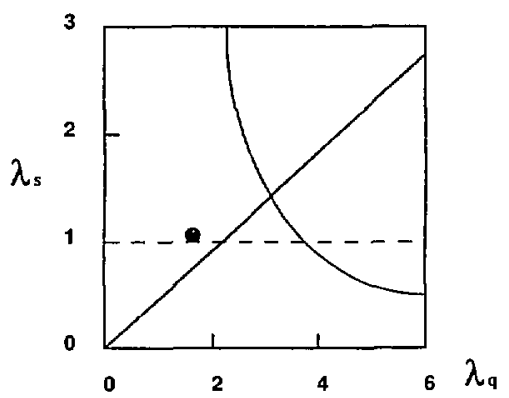

Figure 6: Constraint between the strange-quark fugacity $\lambda_{s}$ and the light quark fugacity $\lambda_{\mathrm{g}}$, choosing fixed ratios of $\mathrm{K}^{+} / K^{-}$and $N \lambda$ (from the E859 experiment) in a themal fireball model. The horizontal dotted line indicates the result expected for QGP. The CERN result is indicated as a solid dot.

b Strange particle phase space is NOT fully occupied (it is also called "partial saturation" of the strangeness phase-space). When produced, strangeness is distributed among the different particles as governed by the size of the accessible phase space.

$c$ fugacity $~$ concentration of quarks 
The straight line represents $\lambda_{S}=0.47 \lambda_{q}$ taken from the kaon ratio and the squared hyperbolad curve reflects the parametrization $\lambda_{s}=17\left(1 / \lambda_{q}{ }^{2}\right)$ deduced from the lambda/antilambda ratio. These lines cross at $\lambda_{s} \sim 1.5$ and $\lambda_{q}$ slightly above 3 . The large value of $\lambda_{q}$ indicates a relatively high baryon density for a mid-rapidity fireball at the AGS, whereas the strangeness fugacity $\lambda_{s} \sim 1.5$ implies $\mu_{s} / T \sim 0.4$ (with $\sim 20 \%$ error bar). The latter is considerably different from the CERN result $\lambda_{s} \sim 1$ (and $\mu_{\mathrm{s}} \sim 0$ ), as will be discussed later in the paper.

As a consistency check, one would like to independently measure relative yields of $\mathrm{p}$ and $\mathrm{p}$ and compare $\mathrm{p} / \mathrm{p}$ ratios $^{\mathrm{e}}$ with the value calculated indirectly from the kaon and lambda ratios $f$. $p / p$ (and $\Lambda / \Lambda$ ) ratios are very interesting, but difficult to specify precisely. Since protons are predominantly from participant nuclei and not from produced particles, so their rapidity distribution is very different in shape compare to a $\bar{p}$. Thus $\mathrm{p} / \mathrm{p}$ has a strong rapidity dependence, even at mid-rapiditys .

However one can calculate the $p / p$ ratio at a fixed rapidity (very small rapidity interval), e.g. at $y=1.3$ ( mid-rapidity) dN/dy ( $p) \sim 10^{-2}$ and $d N / d y(p)$ is $\sim 20$, thus $p / p$ at $y=1.3$ amounts to about $(0.5 \pm 0.2) \times 10^{-3}$, which agrees within two standard deviations with the E859 $\mathrm{p} / \mathrm{p}$ value extracted from the $(\Lambda / \Lambda) /\left(\mathrm{K}^{+} / \mathrm{K}^{-}\right)$ratio $^{\mathrm{h}}$.

Our picture is still incomplete. The key signature for the quark-gluon plasma formation is the saturation of the strange quark phase space, due to the shorter time scale expected in the QGP ${ }^{1}$. Assessment of the degree of strangeness saturation would require additional experimental information, e.g. measurements of the $\Lambda / p$ ratio. This is not available presently from existing AGS experiments. We will come back to the more detailed discussion of the strangeness saturation factor after visiting CERN results on strangeness.

\section{CERN RESULTS ON STRANGENESS AND SOME ATTEMPTS AT ITS INTERPRETATION}

A vast body of data now exists on the spectra of mesons, baryons and hyperons produced in the collisions initiated by sulphur nuclei at CERN energy. To illustrate the quality of the data and the sophistication of the experiments, I have chosen, as an example, the transverse mass spectra from the NA35 experiment presented in Fig.7. The inverse slope parameters of all hadrons except pions exhibit "thermal" behavior. Their values are $210 \pm 20 \mathrm{MeV}$, which is surprisingly high for a hadronic system. Remarkable consistency between transverse mass distribution of neutral and averaged $\left(0.5 \times\left(\mathrm{K}^{+}+\mathrm{K}^{-}\right)\right)$ charged kaons produced in $\mathrm{S}+\mathrm{Ag}$ collisions indicates the quality of the analysis $\mathrm{i}$.

Equally excellent results emerged from CERN experiments also on strange particle production. The first messenger of the unusual behavior arrived in 1988 (two years after the first heavy ion beams at CERN) with the NA35 report on enhanced $\Lambda, \Lambda$ and $\mathrm{K}^{\circ}$ yields in $S+S$ collisions at $200 \mathrm{GeV} / \mathrm{c}^{11}$ These early results have shown that the yields of strange particles relative to the yield of non-strange particles produced in central $S+S$ collisions are higher than the corresponding yields in nucleon-nucleon interactions at the same energies. This observation and the lack of strangeness enhancement in nucleonnucleus collisions ${ }^{12}$ ruled out the interpretations of models based on the superposition of $\mathrm{p}-\mathrm{p}$ or $\mathrm{p}-\mathrm{N}$ collisions.

$d$ here a $(1 / x)^{2}$ dependence

e Ratios involving protons should be taken with a grain of salt sinee experimental values need to be corrected due to contamination by cold projectile and target spectator protons. This task is ussually difficult.

f $\overline{\mathrm{p}} / \mathrm{p}$ ratios can be expressed by the double ratio of $(\bar{N} \Lambda) /\left(\mathrm{K}^{+} / \mathrm{K}^{-}\right)$.

g Note that the slopes of protons and antiprotons are higher than $\pi$ 's and K's. This suggests that at the AGS, they may be freezing in different conditions (the same situation is seen in the CERN data).

$h$ In these calculations the resonance decays, which roughly double the yields of both protons and hyperons. are not taken to the account - therefore above relations hold only approximately.

i Buth data sets were obtained with different detection techniques. 
7

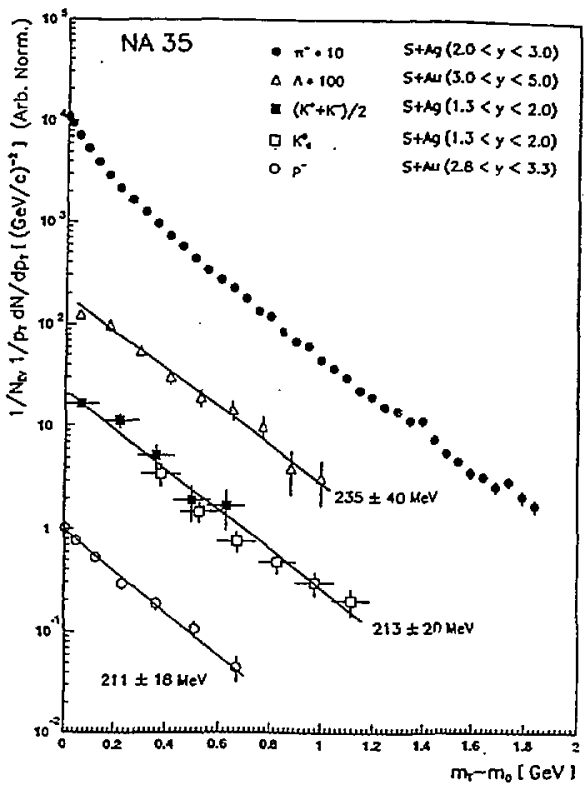

Figure 7: $\mathrm{M}_{\mathrm{t}}$ spectra of different particles in $\mathrm{S}+\mathrm{Au}$ and $\mathrm{S}+\mathrm{Ag}$ collisions at $200 \mathrm{GeV} / \mathrm{c}$ (NA35 experiment).
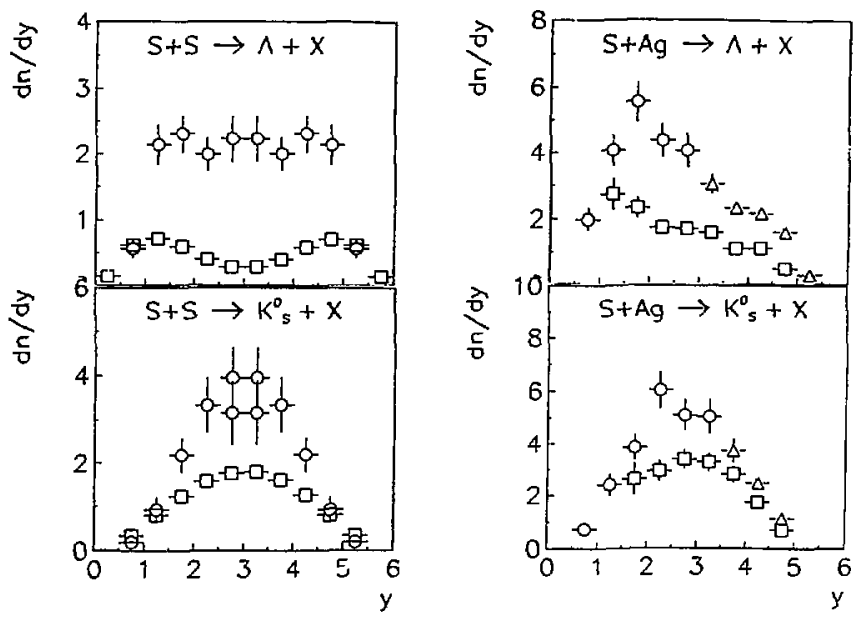

Figure 8: $\Lambda$ and $\mathrm{K}^{0}$ rapidity distributions in $\mathrm{S}+\mathrm{S}$ and $\mathrm{S}+\mathrm{Ag}$ collisions at $200 \mathrm{GeV} / \mathrm{c}$ (NA35). 
New results from the NA35 collaboration have shown that the enhanced strangeness production also persists in central $S+A g$ collisions at $200 \mathrm{GeV} / \mathrm{c}^{13}$. Fig. 8 shows the $\Lambda$ and $\mathrm{K}^{\circ}$ rapidity spectra (over full phase space) in collisions of S on Ag (a asymmetric system) and $S$ on $S$ (a symmetric system).

A comparison of the $\Lambda$ distribution in $S+A g$ and $S+S$ collisions and a scaled distribution of minimum bias $p+S$ interactions for $S+A g$ and $p+p$ interactions for $S+S$ (with the scale factor adjusting the multiplicities of negative charged hadrons) shows that the production is not only enhanced, but that this enhancement is most pronounced around midrapidity. The $\Lambda$ rapidity density at midrapidity and below increases with increasing target mass, whereas for $y>4$, almost no dependence is observed. The production of $\mathrm{K}_{S}{ }^{\circ}$ in $S+A g$ and $S+S$ collisions is enhanced by factor of 1.7 compared to the scaled yield of $\mathrm{p}+\mathrm{S}$ (for $\mathrm{S}+\mathrm{Ag}$ ) and $\mathrm{p}+\mathrm{p}($ for $\mathrm{S}+\mathrm{S}$ ). The charged kaons show a similar trend. Antilambda production is concentrated at midrapidity and the trend seems to be very much like the one seen in $\Lambda$ 's, although the emor bars are still very large ${ }^{13}$. It is quite surprising tiat the strength of this effect is almost the same in all cases $(\Lambda, \Lambda$ and kaons) in both $S+S$ anı $S+A \Sigma$ collisions, since one would expect that strangeness enhancement more pronounced in collisions of heavier systems.

Consistently, the strangeness suppression factor which was used to estimate the deviation from flavor symmetry at the quark level in the final state ${ }^{14}$ has been found to be similar in $S+S$ and $S+A g$ collisions and inly half as strong as in nucleon-nveleon and nucleon-nucleus collisions.

Other new results from the NA2s experiment which address the systematic study of inverse slope parameters in $\mathrm{p}+\mathrm{S}$ a $\mathrm{d} \mathrm{S}+\mathrm{A}$ interactions at $200 \mathrm{GeV} / \mathrm{c}$ are presented in Fig.9.

$T$ [MeV]

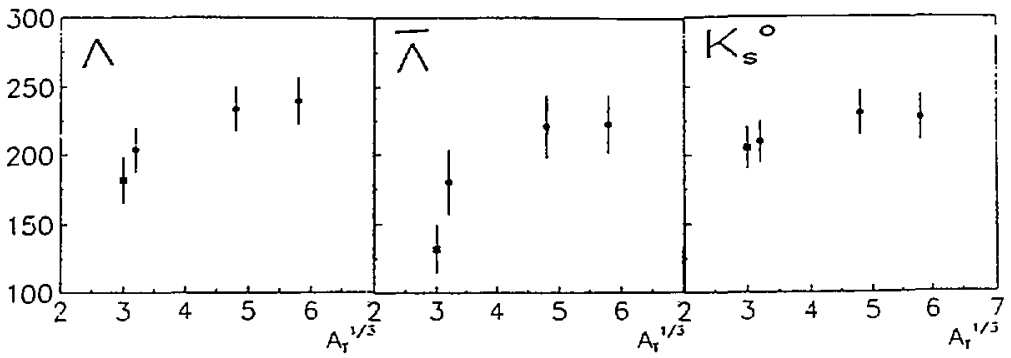

Figure 9: Inverse slope ("temperature" parameier) for $\Lambda, \bar{\Lambda}$ and $K^{\circ}$ in $p+S$ and $S+A$ collisions at 200 $\mathrm{GeV} / \mathrm{c}$ (NA35).

The inverse slope for $\Lambda, \bar{\Lambda}$ and $K_{s}{ }^{0}$ is higher in $S+A$ than in $p+S$ and further increases with the size of the target nucleus.

The NA35 strange particle ratios, summarized in table 1, were used to extract the quark (baryon number) and strange quark fugacities and the strange quark phase-space occupancy at the freeze-out temperature.

Within the generalized thermal model approach 8.10 , discussed in the previous section, the strange quark chemical potential was found to be consistent with zero, and the strange quark abundance was found to be close to the full strangeness saturation as would be expected for the deconfined phase ${ }^{14}$. The same analysis applied to nucleonnucleon interactions at $200 \mathrm{GeV} / \mathrm{c}$ and to nucleus-nucleus collisions at the AGS (see previous paragraph) yields significantly different freeze-out parameters, in particular regarding the degree of strangeness saturation $\mathrm{j}$. The freeze-out temperature obtained

j In p-p only, at AGS the degree of strangeness saturation can not be assessed. 
from the particle yields is consistent with the slopes of transverse mass distributions, which support the notion of a common chemical and thermal freeze-out required in explosive disintegration of a high entropy source ${ }^{14}$. An extention of this model avoids the strong assumption of thermal equilibrium (essential at the present stage) in favor of admitting an additional parameter ${ }^{15}$. Consequently, a preliminary estimate suggest that the strangeness phase space saturation, $\gamma$, will decrease in value by about $60-70 \%$.

Table 1: Strange particle ratios from NA35 experiment.

\begin{tabular}{|c|c|c|c|}
\hline $\begin{array}{c}\text { Multiplicity } \\
\& z \text { Ratio }\end{array}$ & $\begin{array}{c}\text { S-S } \\
4 \pi\end{array}$ & $\begin{array}{c}\text { S-S } \\
\text { sidrap. }\end{array}$ & $\begin{array}{c}N-N \\
4 \pi\end{array}$ \\
\hline$\frac{\pi}{\Lambda}$ & $0.18 \pm 0.05$ & $0.28 \pm 0.1$ & $0.135 \pm 0.055$ \\
$\frac{K^{+}}{K^{-}}$ & $1.8 \pm 0.1$ & $1.45 \pm 0.4$ & $1.4 \pm 0.2$ \\
$\frac{\Lambda}{p-p}$ & $0.41 \pm 0.08$ & $0.64 \pm 0.2$ & $0.11 \pm 0.02$ \\
\hline
\end{tabular}

Reports from NA36, another large CERN experiment designed to study stran:geness, support the NA35 results. Fig. 10 presents the lambda yields as a function of centrality in $S+S$ collisions measured by the NA35 and NA36 experiments (the acceptance of NA36 was adjusted to match that of NA35).

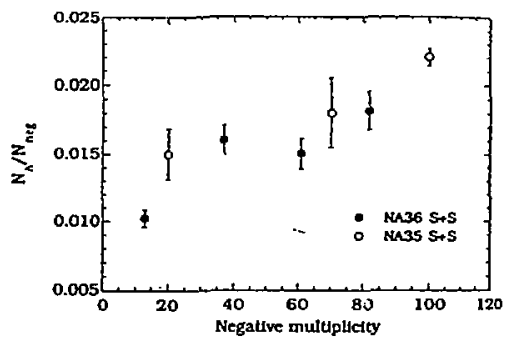

Figure 10: Lambda yields as a function of centrality in $\mathrm{S}+\mathrm{S}$ collisions at $200 \mathrm{GeV} / \mathrm{c}$ (NA35 and NA36).

The comparison shows not only that the production of $\Lambda$ 's in both experiments grow faster with centrality (measured by the multiplicity of negative particles, mostly pions) than the production of negative particles, but also good agreernent between these two experiments. The NA36 results, with sulphur collisions on heavier targets, show a monotonically incraasing strangeness yield that saturates at high multiplicities, where all the projectile nucleons are involved in the collision, with centrality. Analysis shows that the strangeness production in $\mathrm{S}+\mathrm{Pb}$ is almost a factor of two higher than in $\mathrm{p}+\mathrm{Pb}^{16}$.

In addition to $\Lambda, \Lambda$ and $K^{\circ}$ yields, the NA36 TPC detected of $\Xi^{-}$and $\Xi^{+}$hyperon decays. This data is of great importance since the information of singly strange particles alone does not unambiguously distinguish between the hadronic gas and the quark-giuon plasma phase scenarios. The relative abundances of strange (antistrange) baryons with the same or different strangeness content contain a unique characterization of a new form of matter. The particle ratios, after subtraction of the $\Xi^{-}, \Xi^{+}$contribution to the $\Lambda, \bar{\Lambda}$ yields, are listed in Table 2. These ratios are also plotted in Fig.11 and compared with a previously published compilation of measurements ${ }^{17}$. The NA36 results indicate that the enhancement in the $\Xi+1 \bar{\Lambda}$ ratio is at most a factor $2-3$ over the $p+p$ results. The particle ratios of Table 2 allow one to extract ${ }^{18}$, within the same, already discussed, general 
thermodynamical scenario, values: $\mu_{B} / T=1.73 \pm 0.15, \mu_{s} /=0.03 \pm 0.06$ and $\gamma_{s}=0.38 \pm$ 0.04 .

Table 2: Strange particle ratios and their acceptance intervals from NA36 experiment.

\begin{tabular}{lll}
\hline & Paricle natio & Acceptance \\
\hline$\Lambda / \Lambda$ & $0.117 \pm 0.01:$ & $2.0<y<2.5$ \\
$\Xi \pm / \Xi^{-}$ & $0.276 \pm 0.108$ & $2.0<y<2.5$ \\
$\Xi-1 /$ & $0.066 \pm 0.913$ & $1.5<y<2.5$ \\
$\Xi \pm / A$ & $0.127 \pm 0.022$ & $2.0<y<3.0$ \\
\hline
\end{tabular}

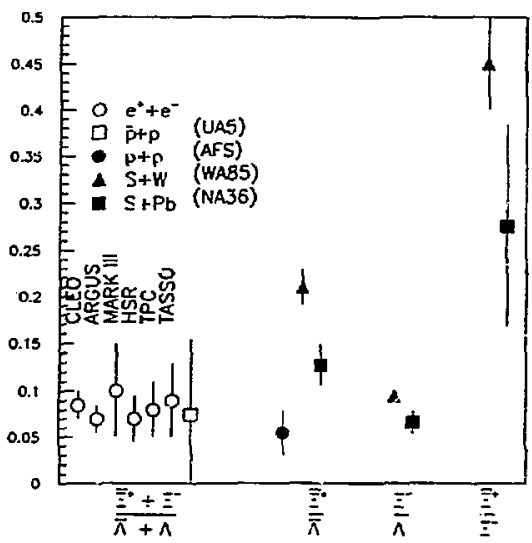

Figure 11: A summary of the world data on various particle ratios involving $\Lambda, \bar{\Lambda}, \Xi^{-} \cdot$ and $\Xi^{+}$.

Difference in values of chenical potentials measured by various CERN experiments can be interpreted as a reflection of different degrees of centrality of the collisions. However, $\mu_{s}$ is small and compatible with zero (and consistent with NA35 measurements). This implies that the system formed near central rapidity contains $1.2 a r l y$ equal number of strange and antistrange quarks. To extract a vaiue for the strange quark saturation factor, $\gamma_{s}$, the ratio of doubly- to singly-strange particle species was uredk. The calculated $\gamma_{s}$ value of $0.38 \pm 0.04$ differs from that expected for the plasma case $\left(\gamma_{s} \sim 1\right)$, where the strange quarks would be close to their equilibriura abundance.

An alternative approach, also founded on thermodynam isal arguments, was pr sposed by Cleymans and Satz. They have shown that in a model of an ideal gas composed of hadrons and known hadronic resonances at fixed temperatures and baryon density, which is assumed to be in equilibrium with zero ne $i$ strange quark density, one can tabulate the production rates of strange particles as a function of $T$ and $\mu_{B}$. The measured ratios and their experimental uncertainties can then be used to establish an allowed region of the $T$ $\mu_{B}$ plane, which is necessary to explain the observed yields of $\Lambda, \bar{\Lambda}, \Xi^{-}$and $\Xi^{+}$. The details of this approach could be found in J.Cleymans contribution to this proceedings.

Slightly different ratios ${ }^{19}$ are reported by the CERN WA85 experiment (see the triangles in Fig.11), presumably due to the d: 'ferent phase space coveragel and, therefore, diffierient values of $\mu_{\mathrm{B}}$. However the $\mu_{\mathrm{S}}$ value remains consistent with zero.

The rare $\Omega^{-} s$ are of particular interest since they contain three strange quarks; therefore their production by rescattering is practically impossible. The first and only experimental information on omega particles in heavy ion collisions was reported by the WA85 experiment ${ }^{22}$. Ainazingly from a data sample of about 60 million triggers, only $7 \pm$ $3.6 \Omega^{-1} \mathrm{~s}$ and $4.0 \pm 2.0 \bar{\Omega}^{-1} \mathrm{~s}$ survived all cuts applied curing the analysis. The estimated ratio $\vec{\Omega}^{-1} / \Omega^{-}$for central rapidity $(2.5<y<3)$ and high $\mathrm{p}_{\mathrm{t}}\left(\mathrm{p}_{\mathrm{l}}>1.6 \mathrm{GeV} / \mathrm{c}\right)$ is $0.57 \pm 0.41$. This ratio is not yet corrected for possible differences in acceptance and efficiency for $\Omega^{-}$'s and $\bar{\Omega}^{-’}$ s.

$k \gamma_{s}{ }^{2}$ is giver by product of the $\equiv-/ \Lambda$ and $\equiv+/ \bar{\Lambda}$ ratio.

1 WA85 experiment acceptance covers only high $\mathrm{p}_{\text {l }}$ region of the phase space $\left(\mathrm{p}_{\mathrm{t}}>1 \mathrm{GeV} / \mathrm{)}\right)$ 
I will stop the reviewing of experimental results hers and conclude that the enhancement of strangeness at CERN has become certain.

But what about theory? Art there any expianations of the olsserved phinomena within available models?

We have seen that some of the thermodynaitical models did manage to describe the measured strange particle ratios fairly well. Let us now examine microscopic models. First of all, there is no ARC type model for CERN energies. All models in which the produced particle system has passed through a phase of thermal equilivium 21.22 do not predict enhanced population of strange particles observed by the CERN cxperiments. More successful in describiilg strangeness enhancement in heavy ion collisions at CERN energies appear to be nor-equilibrium models based un a string picture which employ new mechanisms. The formation of "double sirings" connected to the same leading quarks to enharce the prodisction of baryons containing strange quarks was introduced in the VENIJS model 23 . In the RQMD mode!, a mechanisn of string fusion into "color ropes" 24 . which break faster, allowed more frequent producion of strange quarks and diquarks resulting in enhinced $\bar{\Lambda}$ and $\bar{p}$ production. Very recent, and still preliminary, NA3.5 results on $\rho$ and $A$ yields may help shed some light. The measured ratio $\Lambda$ p near mirirapidity is approximately 1 and is thereiore significantly larger than the corresponding ratio observed in p+o collisions and minimum bias $p+A$ interactions and shows a slight dependence with ưse larget mass in $S+A$ collisions. Fig. 12 summarize the rapidity density of aluipretons at midrapidity observed in central $S+A$ collisions at 200 $\mathrm{GeV} / \mathrm{c}$.

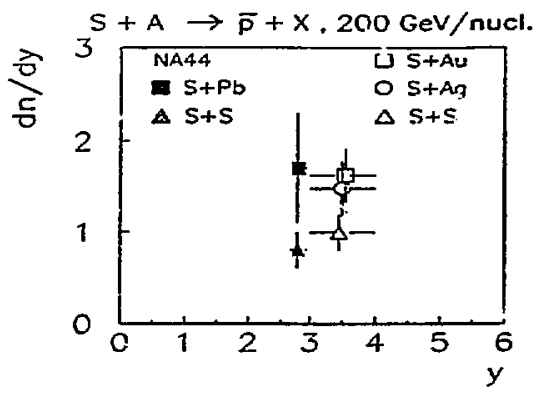

Figure 12: Rapidity density at midrapidit/ of antiprotons (primondial and from decays) produced in central $\mathrm{S}+\mathrm{A}$ collisions at $200 \mathrm{GeV} / \mathrm{c}$ (NA35 experiment).

However all of these new mecharisms, which allowed mcjels to come closer to experimental measurements, also affect pion yields. At this moment, there are no models which would provide satisfactory simultaneous description of strangeness and pion production at CERN energies.

\section{INSTEAD OF CONCLUSIONS}

The local thermal equilibrium model, with allowance for only partial saturation of the stringeness phase space, lets us study the properties of high energy nuclear collisions based on the analysis of the particle multiplicities. Table 3 shows the chemical freeze-rut parameters for central collisions at CERN and AGS energies. All three CERN experiments are characterized by vanishing strange quark chemica: potential at large baryon density combined wich a large degree of strangeness saturation, which differs significantly (by more than $4 \sigma$ ) from $\mu_{s}$ at the AGS, where baryon chemical potential is more than two times larger than at CERN. 
Table 3: Comparison of experimental values of thermal parameters.

\begin{tabular}{lllll} 
& AGS & CERN-WA85 & CERN-N 36 & CERN-NA35 \\
\hline$\mu_{\mathrm{s}} \mathrm{T}$ & $0.54 \pm 0.11$ & $0.03 \pm 0.05$ & $0.03 \pm 0.06$ & 0.025 \\
$\mu_{\mathrm{q}} \mathrm{T}$ & $1.3 \pm 0.1$ & $0.39 \pm 0.04$ & $0.57 \pm 0.05$ & 0.48 \\
$\gamma_{\mathrm{s}}$ & & $-50 \%$ & $\sim 40 \%$ & $\sim 100 \%$
\end{tabular}

These numbers invite some speculations: CERN results could be seen as consistent with a scenario of QGP formation followed by a sudden disintegration; $\mu_{\mathrm{s}}=0$ was found despite the expectations that in the hadronization process of QGP the memory about deconfinement will be erased and hadronic particles will reflect conditions of an equilibrates hadronic gas. Interpretation within the hadronic gas scenarios require extremely long fireball lifetimes in order to reach the measured degree of strangeness saturation ${ }^{\mathrm{m}}$. In view of the present results, one can not yet exclude the possibility of QGP formation at the AGS. However, the dramatic difference in strangeness chemical potentials suggests that, if it is formed, it must be much slower than at the SPS. Comparison of baryon chemical potentials is complicated by different impact parameters and sizes of the systems measured at both energies.

However, granting the fact that the observed particle ratios for hyperons and antihyperons were reproduced in the scenario of a hot hadronic system freezing out rapidly into the final state, one has to go back and verify the underlying assumptions. This is not going to be easy at all. Therefore, one has to be very careful with any speculative conclusions until more data are available. The future experiments will provide, hopefully, the information on the dependence of "thermal" parameters on the masses of the colliding systems, their impact parameters, and energyn ${ }^{n}$. Such a systematic study might allow us to understand the properties of the "macroscopic" high energy density system created in the collisions. In particular, it will be important to determine whether a change of the strangeness saturation factor and chemical fugacity is gradual with $\sqrt{S}$ or sudden as is characteristic of color deconfinement.

Furthermore, the measurements of other strange and non-strange particle ratios are necessary to constrain the final state better, since presently there are only three independent particle ratio results available ${ }^{\circ}$.

On the theoretical side, let us hope that the present results provide sufficient motivation for working out a consistent dynamical non-equilibrium hadronization scenario for a quark-gluon plasma.

\section{ACKNOWLEDGMENTS}

This work was supported in part by the Director, Office of Energy Research, Division of Nuclear Physics of the Office of High Energy and Nuclear Physics of the U.S. Department of Energy under contract DE-AC03-76SF00098.

\section{REFERENCES}

1. P.Koch, B.Muller, J.Rafelski, Phys.Rep.142, 167 (1989)

2. M.Gazdzicki and NA35 Collaboration, Nucl.Phys.A 498 (1989) 375c.

3.A.Bamberger et al.,Phys.Lett.B 184(1987)271; J.Bachler et al.,Z.Phys.C 52 (1991) 239.

4. Mi.Sarabura and E802 Collaboration, Nucl.Phys.A 498 (1989) 409c.

$\mathrm{m}$ No signs for such long hadronic lifetime are seen in the data, e.g. HBT analysis.

$n$ This calls for Lead-Lead or Gold-Gold collisions at different energies, and NOT only at the highest one.

${ }^{\circ}$ which one tries to reproduce with three parameters. 
5. M.Jacob, Proceedings of the Fifth International Conference on Nucletis-Nucleus Collisions, Taormina, Italy, 30 May - 4 June, 1994.

6. S.E.Eiseman et al., Phys.Lett.B 325(1994)322

7. J.Rafelski and M.Danos, Phys.Rev.C - in press.

8. J.Letessier, J.Rafelski and A.Tounsi - preprints PAR/LPTHE/94-15, PAR/LPTHE/9423, and CERN-TH 7304/94, Phys.Lett.B 328(1994)499.

9. e.g. E.Schnedermann, J.Sollfrank and U.Heinz, in: Particle Production from Highly

Excited Matter, Plenum Press, New York, 1993, p. 175; N.Davidson, H.Miller,

R.Quick and J.Cleymans, Phys.Lett.B 255(1991)105; J.Cleymans and H.Satz,

Z.Phys.C 57(1993)135; J.Cleymans, K.Redlich, H.Satz and E.Suhonen, Z.Phys.C 58(1993)347

10. J.Letessier, A.Tounsi, U.Heinz, J.Sollfrank and J.Rafelski, preprint PAR/LPTHE/9227 and TPR-92-28, submitted to Phys.Rev.D.

11. M.Gazdzicki and NA35 Coll., Nucl.Phys.A 498(1989)375c.

12. H.Bialkowska et al., Z.Phys.C 55(1992)491.

13. D.Roh ch and NA35 Coll.,Nucl.Phys.A 566(1994)35c, M.Gazdzicki and NA35 Coll., Nucl.Phys.A 566(1994)503c.

14. J.Sollfrank, M.Gazdzicki, U.Heinz, J.Rafelski, Z.Phys.C 61(1994)659.

15. J.Rafelski - Private communication.

16. E.Andersen et al., Phys.Lett.B 294(1992)127.

17. S.Abatzis et al., Phys.Lett.B 270(1991)123, and references therein.

18. E.Andersen et al., Phys.Lett.B 327(1994)433.

19. D.Evans et al., Nucl.Phys.A 566(1994)225c.

20. Abatzis and WA85 Coll., Nucl.Phys.A 566(1994)49lc.

21. H.W.Barz et al., Nucl.Phys.A 525(1991)435c.

22. J.Cleymans, H.Satz, Z.Phys.C 57(1993)135, J.Badier et al., Phys.Lett.B 122(1983) 441.

23. K.Werner, J.Aichelin, Phys.Lett.B 308(1993)372.

24. H.Sorge et al., Phys.Lett.B 289(1992)6, H.Sorge et al., Preprint LA-KR-92-1078. 